\title{
Mirrored Plasmonic Filter Design via Active Learning of Multi-Fidelity Physical Models
}

\author{
Jialin Song ${ }^{*}$, Yury S. Tokpanov ${ }^{*}$, Yuxin Chen ${ }^{\dagger}$, Dagny Fleischman ${ }^{\dagger}$, \\ Katherine T. Fountaine ${ }^{\ddagger}$, Yisong Yue ${ }^{\dagger}$, Harry A. Atwater ${ }^{\dagger}$ \\ ${ }^{*}$ Division of Engineering and Applied Sciences, California Institute of Technology, 1200 E. California Blvd., \\ Pasadena, CA 91125, USA \\ ${ }^{\ddagger}$ NG Next, Northrop Grumman Corporation, One Space Park, Redondo Beach, CA 90278, USA
}

\begin{abstract}
We designed mirrored plasmonic filters using an advanced active machine learning algorithm that efficiently explores multiple physical models with different approximation fidelities and costs. This method is applicable to a variety of nanophotonics optimization problems. (C) 2020 The Author(s)
\end{abstract}

OCIS codes: 220.4830, 130.7408 .

Introduction Plasmonic devices look very promising as a platform for designing ultra-compact integrated narrow-band photonic filter. Recently Fleischman et al. [1] demonstrated a novel design for optical and nearinfrared color filters with high spectral resolution (FWHM $<31 \mathrm{~nm}$ ) and subwavelength dimensions. In these mirrored filters a single plasmonic slit is coupled to an in-plane Fabry-Perot resonator by metal mirrors, comprising a plasmonic cavity (see Fig. 1a). Combining such individual filters into pixel arrays enables compact image sensors for multispectral and hyperspectral applications (see Fig. 1b).

Designing such mirrored plasmonic filters sets up a non-trivial optimization challenge. Due to the interplay between several physical resonances, the optimization landscape is non-convex with many local minima (see details in [1]). In addition, simulating a particular configuration of a mirrored plasmonic filter requires a computationallyexpensive numerical procedure, which limits the applicability of purely stochastic optimization approaches. We seek intelligent methods which execute global, derivative-free search, with an efficient adaptive strategy that minimizes the overall computational cost of the numerical solver.

Here we present an application of an in-house developed Bayesian optimization strategy based on multi-fidelity Gaussian processes (GP) [2] to this nanophotonics design problem. Our simulation setup allows us to utilize low fidelity physical models (e.g., numerical simulators with coarse geometric mesh size, and/or low simulation time) to aid with the optimization of the (high fidelity) target physical system. We compare our method with other GP approaches and an algorithm commonly used in nanophotonics community — Particle Swarm Optimization.

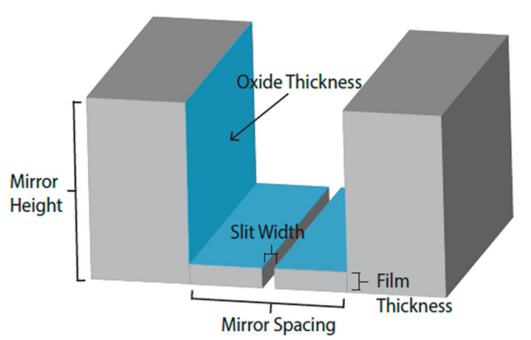

(a)

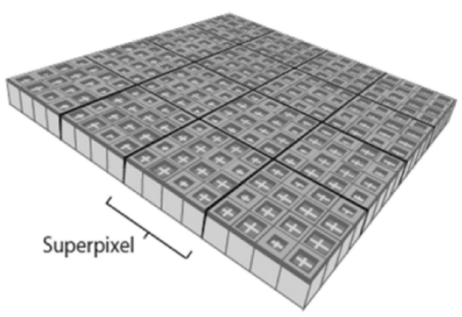

(b)

Fig. 1: a) Mirrored plasmonic filter and its optimization parameters. b) Concept of a hyperspectral imaging sensor, based on mirrored plasmonic filters. Reprinted with permission from [1]. (C2019 American Chemical Society.

Active learning of multi-fidelity physical models We briefly describe a novel strategy proposed in [2] - a mutual information based multi-fidelity Gaussian process (GP) optimization algorithm (MF-MI-Greedy). It consists of two components: an exploratory procedure to gather information about the target (i.e., the highest fidelity) function via querying lower fidelity functions; an exploitative procedure to optimize the target level fidelity with the previously gathered information. MF-MI-Greedy considers an information-theoretic criterion for greedily choosing low fidelity queries. At the end of the exploration phase, MF-MI-Greedy updates the posterior distribution of the joint GP using the full observation history, and searches for a target fidelity action via the (single-fidelity) GP

\footnotetext{
* These authors contributed equally to this work.
} 


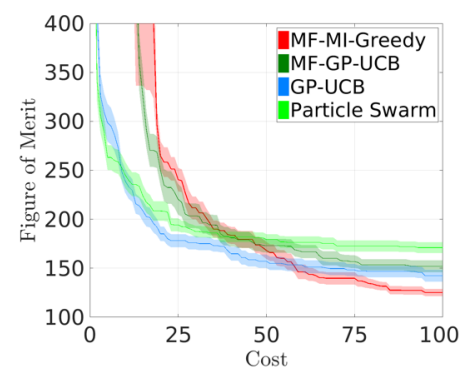

(a)

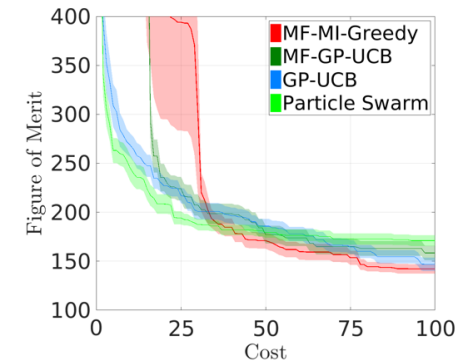

(b)

Fig. 2: Results of optimization trials, in which fidelity level was controlled via: a) mesh size, b) simulation time. Smaller Figure of Merit (FOM) is better. Every method is run 20 times and we plot the mean plus/minus one standard error in the figures.

optimization subroutine, that could be any off-the-shelf Bayesian optimization algorithm. We use a common approach, namely GP-UCB [3], in our implementation. Different from the previous exploration phase, which seeks an informative set of low fidelity actions, the GP optimization subroutine aims to trade off exploration and exploitation on the target fidelity, and outputs a single action at each round. MF-MI-Greedy then proceeds to the next round until it exhausts the preset budget, and eventually outputs an estimator of the target function optimizer.

Setup for computational experiments We use Lumerical commercial implementation of the finite-difference time-domain (FDTD) method to simulate the transmission spectra of our devices. We extract scalar measures of the goodness of the spectrum such as transmission peak amplitude, its offset from designed wavelength, full-width half-maximum, signal-to-noise ratio, and combine them into one weighted Figure of Merit (FOM). Using this FOM, the evolution of the design can then be pursued as a minimization problem over a 5-dimensional geometric parameter space (see Fig. 1a). By varying the geometrical mesh size and the time-domain solver total time duration of simulated physical processes we obtained two sets of multi-fidelity data (effectively creating different physical models of our devices), each with three fidelity levels on 4983 designs.

For all experiments, we use a total runtime budget of 100 times the cost of target fidelity function call. Every method is run with 20 trials to compute its mean and standard error.

Compared methods and results We compare with a popular multi-fidelity baseline algorithm, namely MFGP-UCB [4], and the single-fidelity baseline algorithm, GP-UCB [3]. Besides the Bayesian optimization based methods, we also compare with a common heuristic called Particle Swarm Optimization [5], which is inspired by the social behavior of animals and is often used for nanophotonic structure designs.

Fig. 2 shows the results of this experiment. After a small portion of the budget is used in initial exploration, MF-MI-Greedy (red) is able to arrive at a better final design compared with MF-GP-UCB, GP-UCB and Particle Swarm. MF-MI-Greedy tends to have a worse figure of merit in the beginning because initial explorations in the lower fidelity do not yield FOM scores on the target fidelity, so essentially it has a late start in all the plots because it starts querying the target fidelity late. However, the advantage of exploring lower fidelities becomes apparent once the exploitation phase starts in the target fidelity level, as seen by the rapid convergence to low FOM designs.

Conclusion Our results on several pre-collected nanophotonics datasets demonstrate the compelling performance of the multiple-fidelity Bayesian optimization approach. These experiments suggest that there is a great potential in utilizing cheap, multi-fidelity simulations to aid the discovery of the optimal photonic nanostructures.

\section{References}

1. Dagny Fleischman, Katherine T. Fountaine, Colton R. Bukowsky, Giulia Tagliabue, Luke A. Sweatlock, and Harry A. Atwater. High spectral resolution plasmonic color filters with subwavelength dimensions. ACS Photonics, 6(2):332-338, 2019.

2. Jialin Song, Yuxin Chen, and Yisong Yue. A general framework for multi-fidelity bayesian optimization with gaussian processes. In AISTATS, April 2019.

3. Niranjan Srinivas, Andreas Krause, Sham Kakade, and Matthias Seeger. Gaussian process optimization in the bandit setting: No regret and experimental design. In ICML, 2010.

4. Kirthevasan Kandasamy, Gautam Dasarathy, Junier B. Oliva, Jeff Schneider, and Barnabás Póczos. Gaussian process bandit optimisation with multi-fidelity evaluations. In NeurIPS, 2016.

5. James Kennedy and Russel C. Eberhart. Particle swarm optimization. Proceedings of the IEEE International Conference on Neural Networks. Perth, Australia, pages 1942-1945, 1995. 